\title{
Xylomedes coronata (Marseul, 1883), un xylophage polyphage (Coleoptera, Bostrichidae)
}

\author{
par Henri-Pierre ABERLENC* \& Mohand Salah HAMLAOUI** \\ *Cirad Bios, TA A-55/L, Campus de Baillarguet, F-34398, Montpellier cedex 5 <henri-pierre.aberlenc@cirad.fr> \\ **Service de vulgarisation agricole de la commune de M'Cisna/Seddouk, CP 35, 06540, Bejaïa, Algérie \\ <hamlaoui_salah@yahoo.fr>
}

Résumé. - L'aire de répartition connue de Xylomedes coronata (Marseul, 1883) (Coleoptera, Bostrichidae) s'étend du Maroc à Israël. Il peut se développer dans les branches mortes ou vivantes d'essences variées, dont la Vigne, le Laurier-rose, l'Arganier, le Grenadier, divers Prunus, divers Agrumes et l'Olivier. Si les seuls dégâts sérieux sont cités sur Agrumes au Maroc et sur Olivier en Kabylie, il est susceptible de devenir un ravageur n'importe où dans son aire de répartition, surtout à la faveur des déséquilibres écologiques et d'épisodes chauds et secs. Le commerce d'arbres vivants ou de bois est susceptible de lui permettre de coloniser de nouveaux territoires méditerranéens.

Summary. - Xylomedes coronata (Marseul, 1883), a polyphagous xylophagous (Coleoptera, Bostrichidae). The native range of Xylomedes coronata (Marseul, 1883) (Coleoptera, Bostrichidae) expands from Morocco to Israel. This powderpost beetle has multiple hosts including Grapevine, Oleander, Argan tree, Pomegranate, Prunus, Citrus, and Olive tree. By completing its entire cycle in dead or living wood, it became a serious pest on Citrus in Morocco, and on Olive tree in Kabylie. The pest status varies along its native range and depends on several biotic factors, such as ecological disturbance, temperature and dryness. Other Mediterranean areas are threatened by global trade of timber and living trees.

Keywords. - Pest, potential invasive species, climatic change, dryness, Olea europaea, Citrus, Vitis, Argania spinosa, Punica granatum, Prunus, Mediterranean area.

Le récent catalogue de BOROWSKI \& WĘGRZYNOWICZ (2007) complète les travaux plus que centenaires de LESNE (1901, 1909), d'une clarté exemplaire, admirablement illustrés et encore irremplaçables pour l'identification des Bostriches.

\section{Genre Xylomedes Lesne, 1902}

Imagos. - Mandibules allongées, pointues, se croisant à l'apex ; hanches antérieures subconiques, fortement saillantes ; tarses intermédiaires et postérieurs plus allongés que les tibias (fig. 1, 5) ; premier article du tarse minuscule, à peine visible ; articles 2-3-4 des tarses avec une touffe apicale très dense de courtes soies dorées sur la face inférieure ; déclivité apicale des élytres régulièrement convexe, sans concavité, ni cornes, ni tubercules (fig. 4, 7) ; apex élytral finement denté près de la suture (fig. 8). Dimorphisme sexuel très marqué : angles antérieurs du pronotum du mâle prolongés par une forte corne orientée vers le bas, munie de dents sclérifiées aplaties en forme de triangles émoussés (fig. 6, flèche droite) ; les deux éperons sclérifiés de l'apex du tibia antérieur du mâle plus forts que ceux de la femelle, l'apical étant recourbé en crochet chez le mâle (fig. 6, flèche gauche); front de la femelle concave, couvert d'une dense pubescence, délimité de chaque côté par une carène prolongée en une corne aplatie orientée vers le haut (fig. 3, flèches).

\section{Xylomedes coronata (Marseul, 1883)}

Imago. - 8-11 mm. Pronotum avec une étroite zone longitudinale médiane lisse à la base et de longues soies dorées dressées sur les côtés, en avant. Ponctuation des élytres constituée de gros points rapprochés, très imparfaitement alignés (fig. 2).

Mâle. - Couleur brun foncé. Taille moyenne un peu plus petite que celle de la femelle. Silhouette plus large que celle de la femelle (fig. 5). Sculpture du pronotum constituée de grains écrasés vers la 
base et de plus en plus fortement saillants vers l'avant (fig. 5-6). Vue de profil, partie dorsale antérieure du pronotum arrondie. Pubescence dorsale faite de soies dorées, peu denses, couchées. Pubescence des sternites abdominaux grise, couchée et assez dense. Front convexe, avec une concavité antérieure, la sculpture faite de grains aplatis (fig. 6), avec en avant et latéralement des poils dressés roux, plus courts que ceux de la femelle. Articles des tarses intermédiaires et postérieurs plus courts que chez la femelle (fig. 5). Suture élytrale à peine en relief au niveau de la déclivité apicale des élytres (fig. 7).

Femelle. - Couleur brun-roux, plus claire que chez le mâle, la déclivité élytrale foncée. Taille moyenne un peu plus grande que celle du mâle. Silhouette plus étroite que celle du mâle (fig. 1). Concavité en avant du pronotum, au-dessus de la tête, plus marquée chez la femelle que chez le mâle (fig. 3, 6). Vue de profil, partie dorsale antérieure du pronotum anguleuse et saillante vers le haut. Yeux davantage saillants que chez le mâle (fig. 3, 6). Elytres glabres, sauf l'apex qui porte quelques soies dressées. Pubescence des sternites abdominaux dorée, peu dense, sauf à l'apex. Front concave, couvert d'une dense et longue pilosité rousse, les poils les plus longs sur les côtés, délimité de chaque côté par une carène saillante au-dessus de l'œil, prolongée vers le haut en une étroite corne aplatie émoussée (fig. 3, flèches). Articles des tarses plus longs que chez le mâle (fig. 1,5). Suture élytrale plus fortement en relief au niveau de la déclivité apicale que chez le mâle (fig. 4).

\section{RÉPARTITION GÉOGRAPHIQUE CONNUE}

Maroc : largement répandu. Algérie : Tell algérien (la région côtière fertile). LallaMarnia, Oran, vallée du Chélif, Affreville, Littré, la Chiffa, Palestro, Azazga (Lesne, 1909), Kabylie (région de Béjaïa). Italie : sud-ouest de la Sicile (BEnfatTo \& Longo, 1986). Tunisie (LÖBl \& SMETANA, 2007). Egypte (LÖBL \& SMETANA, 2007). Israël : "Evolution Canyon", lower Nahal Oren (CHIKATUNOV et al., 2004.).

\section{UN RAVAGEUR DE L'OLIVIER EN KABYLIE}

Xylomedes coronata fait de sérieux dégâts sur l'Olivier en Kabylie depuis 1995 (HAMLAOUI, 2007). Les larves et les adultes forent des galeries dans des branches de diverses sections, avec une préférence nette pour celles de petit diamètre (attaques maximales entre $0,6 \mathrm{et} 1 \mathrm{~cm}$, plus faibles entre 1 et $1,5 \mathrm{~cm}$, minimales au-delà de $1,5 \mathrm{~cm}$ ). Les jeunes rameaux dépérissent, se dessèchent et deviennent cassants. Les attaques sont très variables d'un plant à un autre. Les jeunes oliviers (moins de vingt ans) sont les plus attaqués, les arbres de plus de trente ans étant presque indemnes. La nymphose commence entre la fin du mois d'avril et le début de mai. L'émergence des adultes se déroule entre le début du mois de mai et la fin du mois de juin. Ils sont actifs jusqu'à la fin du mois d'août. Les imagos sont nocturnes et sont attirés par la lumière. Les femelles pondent en juillet-août. Le stade larvaire commence en août et se prolonge jusqu'au printemps de l'année suivante. Il n'y a qu'une génération par an. Les attaques sont plus fortes par temps chaud et sec $\left(30\right.$ à $35^{\circ} \mathrm{C}$ et hygrométrie de $\left.48-50 \%\right)$ que par temps plus frais et plus humide $\left(25^{\circ}\right.$ et hygrométrie de $\left.60 \%\right)$; elles varient d'une année à l'autre, les années les plus chaudes et les plus sèches étant les plus favorables à la prolifération de Xylomedes coronata. Les traitements chimiques sont non seulement toxiques pour les êtres humains et polluants pour le milieu naturel, mais encore inefficaces, car ils ne peuvent atteindre ni les larves ni les adultes dans leurs galeries : il faut tailler avec soin les arbres et brûler tous les rameaux attaqués.

\section{XYLOMEDES CORONATA EST-IL UN RAVAGEUR IMPORTANT ET POTENTIELLEMENT INVASIF ?}

Polyphage, se développant dans les branches et les branchettes vivantes ou mortes de divers ligneux au sud et à l'est du bassin méditerranéen, Xylomedes coronata a coexisté depuis des millénaires avec l'espèce humaine sans occasionner durablement ni massivement de dégâts à l'Olivier, aux divers Prunus cultivés, aux Agrumes ni à la Vigne. 


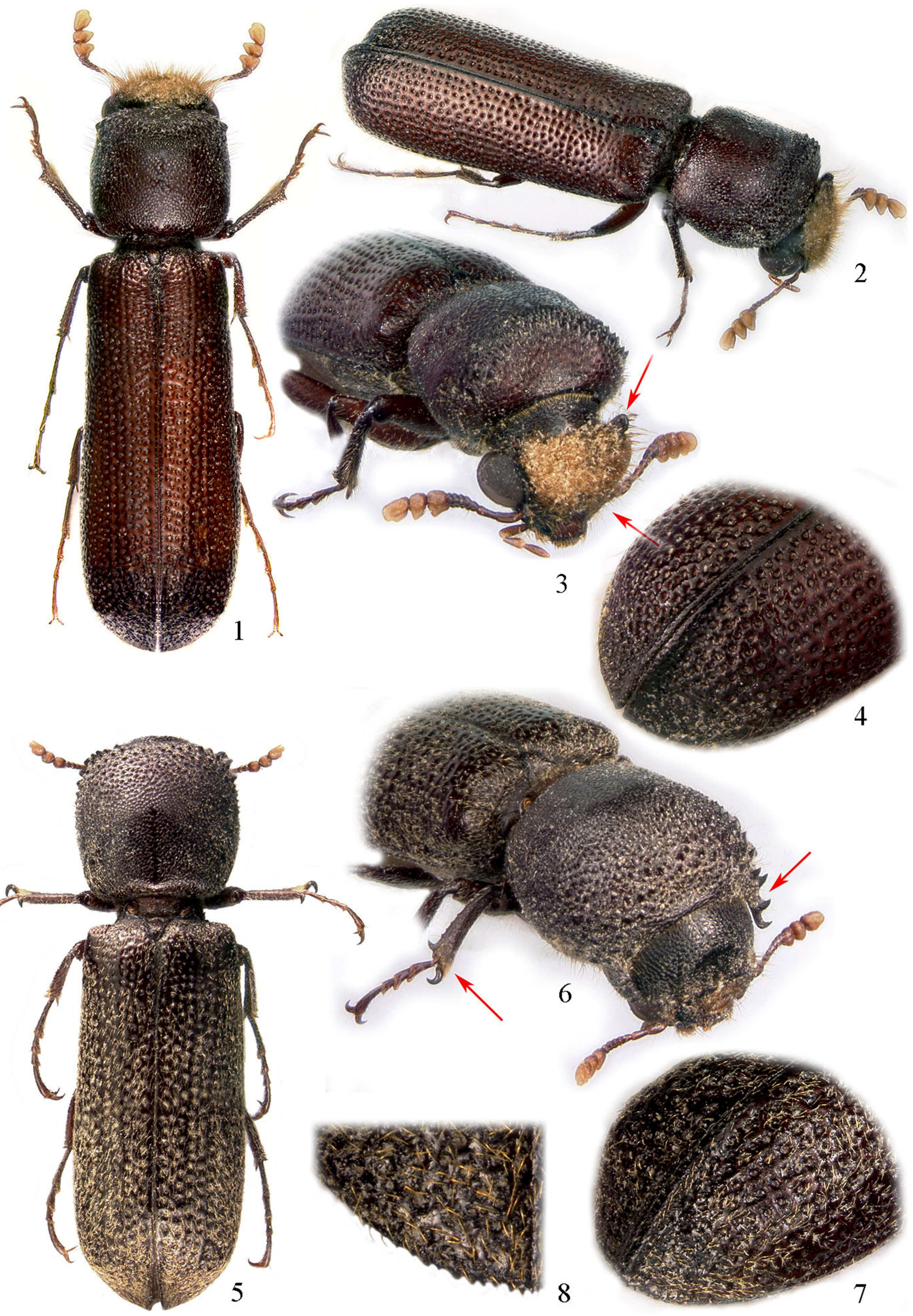

Fig. 1-8. - Xylomedes coronata (Marseul, 1883). - 1-4, Femelle. - 1, Vue dorsale. - 2-3, Vue de trois quarts. - 4, Déclivité apicale des élytres. - 5-8, Mâle. - 5, Vue dorsale. - 6, Vue de trois quarts. - 7, Déclivité apicale des élytres. -8 , Détail de l'apex élytral. 
Tableau I : Plantes-hôtes connues de Xylomedes coronata (Marseul, 1883).

\begin{tabular}{|c|c|c|c|}
\hline Plantes-hôtes & Commentaires & Pays & Auteurs \\
\hline \multirow[t]{2}{*}{ Vigne (Vitis vinifera) } & Sarments. & Algérie & LESNE, 1901 \\
\hline & $\begin{array}{l}\text { "dégâts sensibles" dans une pépi- } \\
\text { nière de Vigne américaine en } 1930 . \\
\text { Galeries creusées par l'adulte dans } \\
\text { de minces branchettes vivantes. }\end{array}$ & Maroc & $\begin{array}{l}\text { BOUHELIER, } 1933 \text {; CHARARAS } \\
\text { \& BALACHOWSKY, } 1962\end{array}$ \\
\hline $\begin{array}{l}\text { Laurier-rose (Nerium } \\
\text { oleander) }\end{array}$ & Bois coupé. & Algérie & LESNE, 1901 \\
\hline Tizra (Rhus pentaphylla) & Elevé dans des branches. & Maroc & BOUHELIER, 1933 \\
\hline \multirow[t]{2}{*}{ Arganier (Argania spinosa) } & $\begin{array}{l}\text { Branches coupées mises en } \\
\text { élevage. }\end{array}$ & \multirow[t]{2}{*}{ Maroc } & BOROWSKI \& MAZUR, 2001 \\
\hline & $\begin{array}{l}\text { "Xylomedes coronata est le plus } \\
\text { redoutable ennemi du bois coupé } \\
\text { d'Arganier ; il se propage par le } \\
\text { biais du bois de taille que les } \\
\text { populations usagères utilisent } \\
\text { pour les clôtures". }\end{array}$ & & M'HIRIT et al., 1998 \\
\hline Grenadier (Punica granatum) & Branches sèches. & Maroc & $\begin{array}{l}\text { PARDO ALCAIDE, } 1955 ; \\
\text { LOPEZ-COLON, } 2000\end{array}$ \\
\hline $\begin{array}{l}\text { Prunier japonais (Prunus } \\
\text { salicina) }\end{array}$ & $\begin{array}{l}\text { Attaques importantes en } 1929,1931 \\
\text { et 1932. Galeries creusées par } \\
\text { l'adulte dans de minces bran- } \\
\text { chettes vivantes. }\end{array}$ & Maroc & $\begin{array}{l}\text { BOUHELIER, } 1933 \text {; CHARARAS } \\
\text { \& BALACHOWSKY, } 1962\end{array}$ \\
\hline $\begin{array}{l}\text { Pêcher (Prunus persica) } \\
\text { et/ou Amandier (Prunus } \\
\text { dulcis) et/ou Abricotier } \\
\text { (Prunus armeniaca) }\end{array}$ & $\begin{array}{l}\text { "Xylomedes sp." provoque le } \\
\text { dessèchement et parfois la rupture } \\
\text { des rameaux. }\end{array}$ & $\begin{array}{l}\text { Maroc et/ou } \\
\text { Tunisie }\end{array}$ & KASSEBEER et al., 1976 \\
\hline Poirier (Pyrus communis) & Simple citation. & Maroc & CHAPOT \& DELUCCHI, 1964 \\
\hline Agrumes divers & $\begin{array}{l}\text { Dégâts sur divers arbres fruitiers } \\
\text { signalés depuis les années } 1930 \\
\text { dans tout le pays mais localement, } \\
\text { sur Agrumes, importants dégâts, } \\
\text { qui peuvent être considérables sur } \\
\text { de jeunes arbres. }\end{array}$ & Maroc & СНAPOT \& DELUCCHI, 1964 \\
\hline Oranger (Citrus sinensis) & Dégâts sporadiques. & $\begin{array}{l}\text { Italie } \\
\text { (Sicile) }\end{array}$ & BENFATTO \& LONGO, 1986 \\
\hline \multirow[t]{3}{*}{ Olivier (Olea europaea) } & Simple citation. & Maroc & $\begin{array}{l}\text { PARDO ALCAIDE, } 1955 \text {; } \\
\text { CHAPOT \& DELUCCHI, } 1964 \text {; } \\
\text { TZANAKAKIS, } 2003\end{array}$ \\
\hline & Simple citation. & $\begin{array}{l}\text { Maroc, } \\
\text { Tunisie }\end{array}$ & KASSEBEER et al., 1976 \\
\hline & $\begin{array}{l}\text { Dégâts sérieux depuis 1995, variables } \\
\text { selon les années. }\end{array}$ & $\begin{array}{l}\text { Algérie } \\
\text { (Kabylie) }\end{array}$ & HAMLAOUI, 2007 \\
\hline
\end{tabular}

Il faut clairement distinguer les dégâts sans réelle gravité occasionnés sur du bois mort coupé, ou sur des plantes sans importance économique, de ceux provoqués sur des branchettes vivantes d'arbres fruitiers en production, qui se dessèchent et se rompent. Et dans ce dernier cas il faut distinguer les dégâts limités dans le temps et l'espace et/ou limités en intensité, de ceux qui s'avèrent localement durables et/ou graves.

Sur l'Arganier, Xylomedes coronata n'est cité que dans du bois coupé et ne semble pas compromettre la production d'huile d'argane. Les cas connus de dommages sérieux sur Vigne et sur Prunier du Japon sont localisés et anciens (années 1930). Les dégâts contemporains sur Agrumes au Maroc et la situation en Kabylie où depuis 1995 X. coronata est devenu un 
ravageur de l'Olivier illustrent que ce xylophage, dont les dégâts sont habituellement anodins ou sporadiques, peut devenir durablement dans certaines régions un ravageur non négligeable.

Dans un monde où les écosystèmes jouxtant les aires cultivées sont anéantis, reculent ou se trouvent gravement déséquilibrés, ne pouvant ainsi plus jouer leur rôle de régulateurs par leurs auxiliaires naturels, Xylomedes coronata est susceptible de devenir un ravageur en tout point de son aire de répartition, du Maroc à Israël, surtout à la faveur d'épisodes chauds et secs : le réchauffement climatique ne peut que favoriser sa prolifération.

Dans un monde où les échanges économiques se sont intensifiés, on ne peut pas non plus exclure que le commerce d'arbres vivants contaminés ou de bois coupé puisse permettre à ce Bostrichide de conquérir de nouveaux territoires méditerranéens. Cette espèce doit donc faire l'objet d'une surveillance attentive.

REMERCIEMENTS. - Nous remercions Mme A. Taghavian et notre collègue et ami T. Deuve pour le prêt de spécimens de Xylomedes coronata de la collection Lesne, déposés au Muséum national d'Histoire naturelle, Paris. Nous remercions notre collègue et ami R. Sforza pour sa traduction.

\section{AUTEURS CITÉS}

BenfatTo D. \& Longo S., 1986. - Beetle pests living on Citrus in Italy. In : Cavalloro R. \& Martino E. D. (eds), Integrated pest control in citrus groves, p. 27-133. Proceedings of the expert's meeting, Acireale, 16-17 March, 1985. Totterdam, Balkema A. A. \& Boston Publishers.

BOROWSKI J. \& MAZUR S., 2001. - Contribution to the knowledge of the Bostrichidae and associated Histeridae of Morocco (Insecta, Coleoptera). Il Naturalista valtellinese - Atti Museo civico di Storia naturale de Morbegno, $12: 69-75$.

BOROWSKI J. \& WĘGRZYNOWICZ P., 2007. - World Catalogue of Bostrichidae (Coleoptera). Olsztyn, Mantis, $247 \mathrm{p}$.

BOUHELIER R., 1933. - Les Insectes xylophages des arbres fruitiers au Maroc. Bostrychides. Revue agricole de l'Afrique du Nord, 28 (713) : 199-201.

CHAPOT H. \& DELUCCHI V. L., 1964. - Maladies, troubles et ravageurs des agrumes au Maroc. Rabat, Institut National de la Recherche Agronomique, 339 p.

Chararas C. \& BALACHOWSKY A. S., 1962. - Famille des Bostrychidae. In : Balachowsky A. S. (éd.), Entomologie appliquée à l'Agriculture. Tome I (Premier Fascicule). Coléoptères, p. 310. Paris, Masson et Cie, $564 \mathrm{p}$.

Chikatunov V., Pavlíček T. \& Nevo E., 2004. - Coleoptera of "Evolution Canyon", Lower Nahal Oren, Mount Carmel, Israel. Part II. Pensoft Publishers, 200 p.

HAMLAOUI M. S., 2007. - Xylomedes sp. (Coleoptera, Bostrychidae), nouvelle espèce nuisible sur olivier en Algérie, dans la région de Bejaïa. Olivae, 108 : 41-52.

Kassebeer H., Keyserlingk N. V., Lange E., Link R., Pollehn E., Zehrer W. \& Bohlen E., 1976. La défense des cultures en Afrique du Nord, en considérant particulièrement la Tunisie et le Maroc. Arbres fruitiers : Pécher, Amandier, Abricotier, Pommier, Poirier. Eschborn, GTZ, 35 p.

LESNE P., 1901. - Synopsis des Bostrychides paléarctiques. L'Abeille, 30 : 73-136, pl. I-IV.

1909. - Révision des Coléoptères de la famille des Bostrychides. $6^{\mathrm{e}}$ mémoire : Dinapatinae et

Apatinae. Annales de la Société entomologique de France, 78 : 471-574, pl. 13-17.

LöBl I. \& SMETANA A., 2007. - Catalogue of Palearctic Coleoptera, Volume 4. Apollo Books, 935 p.

LOPEZ-Colon J. I., 2000. - Los Bostrichidae Latreille, 1802 de la fauna de Marruecos (Coleoptera).

Biocosme Mésogéen, 16 (4) [1999] : 171-221.

M'Hirit O., Benzyane M., Benchekroun F., El Yousfi S.M. \& BendaAnoun M., 1998. L'Arganier, une espèce fruitière-forestière à usages multiples. Sprimont (Belgique), Mardaga, $150 \mathrm{p}$.

PARDo AlCAide A., 1955. - Contribución al conocimiento de la fauna entomológica marroquí. VI. Coleopteros del valle inferior del Uad Muluya. Tamuda, 3 (1) : 39-74.

TZANAKAKIS M. E., 2003. - Seasonal development and dormancy of Insects and mites feeding on olive: a review. Netherlands Journal of Zoology, 52 (2-4) : 87-224. 